\title{
Two new species of the genus Nepalomyia, with a key to species from China (Diptera: Dolichopodidae)
}

\author{
Mengqing Wang \& Ding Yang*
}

Wang, M. \& Yang, D. 2005: Two new species of the genus Nepalomyia, with a key to species from China (Diptera: Dolichopodidae). - Entomol. Fennica 16: 103-108.

The species of the genus Nepalomyia Hollis, 1964 from China are reviewed and keyed. The following two species are described as new to science: Nepalomyia beijingensis sp. $\mathrm{n}$. and $N$. ruiliensis sp. $\mathrm{n}$. The distribution of the genus in China is discussed.

M. Wang \& D. Yang (corresponding author), Department of Entomology, China Agricultural University, Haidian, Beijing 100094, China; Key Lab of Insect Evolution \& Environmental Changes, Capital Normal University, Beijing 100037, China.E-mail: dyangcau@yahoo.com.cn; yangding@cau.edu.cn

Received 2 April 2004, accepted 30 September 2004

\section{Introduction}

The genus Nepalomyia Hollis, 1964 belongs to the subfamily Peloropeodinae. It has been synonymized with Neugrigonella Robinson, 1964 by Runyon \& Hurley (2003). The genus is characterized by the arista arising from the apical concavity of the first flagellomere and hind tarsomere 1 of male bearing 1 basal spur on the posterior surface directed upward. It is known to occur in the Holarctic and Oriental Realms. There is one species known from the Palaearctic, 23 from the Oriental, and four from the Nearctic. The major references dealing with the taxonomy of the genus are Dyte (1975), Hollis (1964), Negrobov (1984), Negrobov (1991), Robinson (1964), Robinson \& Vockeroth (1981), Runyon \& Hurley (2003), Van Duzee (1914), Yang (2001, 2002), Yang \& Saigusa (2000, 2001a-b), Yang, Yang \& Li (1998).

In the present paper, two species of the genus are described as new to science: Nepalomyia beijingensis sp. $\mathrm{n}$. from North China (Beijing), and N. ruiliensis sp. n. from South China (Yun- nan). A key to the Chinese species of the genus is presented. Types of the new species are deposited in the Entomological Museum of China Agricultural University, Beijing.

Abbreviations are as follows: acr $=$ acrostichal, $\mathrm{ad}=$ anterodorsal, $\mathrm{av}=$ anteroventral, $\mathrm{dc}=$ dorsocentral, $\mathrm{h}=$ humeral, ih = inner humeral, LI = fore leg, LII - mid leg, LIII = hind leg, $\mathrm{npl}=$ notopleural, $\mathrm{oc}=$ ocellar, $\mathrm{pd}=$ posterodorsal, $\mathrm{ph}=$ posthumeral, $\mathrm{su}=$ sutural, $\mathrm{v}=$ ventral, and $\mathrm{vt}=$ vertical.

\section{Key to species of Nepalomyia from China (males)}

1. First flagellomere distinctly longer than wide (longa-species group) 2

- First flagellomere wider than long (rarely as long as wide) (henanensis-species group) 5

2. Hind tarsomere 1 shortened and thickened; hypandrium rather large and furcate apically 3

- Hind tarsomere 1 normal 4

3. First flagellomere much elongated, 2.5 times 
longer than wide; basal spur on hind tarsomere 1 furcate. Yunnan

furcata Yang \& Saigusa, 2001

- First flagellomere distinctly elongated, 1.3 times longer than wide; basal spur on hind tarsomere 1 normal. Yunnan

crassata Yang \& Saigusa, 2001

4. First flagellomere with long, lower apical angle (1/3 total length); hypandrium with short apical incision. Yunnan

dentata Yang \& Saigusa, 2001

- First flagellomere with short, lower apical angle (about $1 / 5$ total length); hypandrium with deep apical incision. Shaanxi

longa Yang \& Saigusa, 2001

5. First flagellomere wider than long; 5-6 strong dc

- First flagellomere as long as wide; 4 strong dc. Yunnan daliensis Yang \& Saigusa, 2001

6. $\mathrm{R} 2+3$ and $\mathrm{R} 4+5$ normal

7

- R2+3 and R4+5 distinctly thickened basally. Sichuan, Yunnan

trifurcata Yang \& Saigusa, 2001

7. Thoracic pleura brownish yellow to yellow except pteropleuron or upper portion dark 8

- Thoracic pleura wholly metallic green 13

8. 5 strong dc; thoracic pleura brownish-yellow to yellow, except pteropleuron dark

9

- 6 strong dc; thoracic pleura brownish-yellow to yellow, except upper portion dark

9. Antennal scape and pedicel yellow. Yunnan

- Antenna entirely black.

10. Halter brown with yellow base; hypandrium short and broad, with conical apex. Yunnan flava Yang \& Saigusa, 2001

- Halter yellow; hypandrium with 2 acute denticles. Guizhou

11. acr absent. Yunnan

bidentata Yang \& Saigusa, 2001

pingbiana Yang \& Saigusa, 2001

- acr 4-5 irregularly paired. Yunnan luteipleurata Yang \& Saigusa, 2001

12. Cercus with basal tubercle. Shaanxi tuberculosa Yang \& Saigusa, 2001

- Cercus without basal tubercle. Beijing

13. 5 strong dc beijingensis sp. $\mathrm{n}$.

- 6 strong dc
14. Fore tarsomere 5 dark; halter yellow

- Fore tarsomere 5 pale; halter dark brown with yellow base. Sichuan

pallipes Yang \& Saigusa, 2000

15. Hypandrium trifurcate apically; cercus without distinct basal tubercle, but with short sparse short hairs

- Hypandrium not furcate apically; cercus with basal tubercle bearing short hairs and 2 very long bristles. Sichuan

longiseta Yang \& Saigusa, 2000

16. First flagellomere 0.6 times as long as wide; hypandrium with marginal denticles, middle lobe short with furcate tip in ventral view. Yunnan pallipilosa Yang \& Saigusa, 2001

- First flagellomere nearly as long as wide; hypandrium without marginal denticles, middle lobe long and broad in ventral view. Yunnan ruiliensis $\mathrm{sp} . \mathrm{n}$.

17. First flagellomere with weak lower apical angle

- First flagellomere with distinct lower apical angle

18. Hind tibia with 2 ad

- Hind tibia with $1 \mathrm{ad}$; cercus with long geniculate basal process. Shaanxi, Yunnan zhouzhiensis Yang \& Saigusa, 2001

19. Hind tibia with $3 \mathrm{pd}$

- Hind tibia with 2 pd

20. Hypandrium without long lateral lobes; cercus without basal tubercle. Henan henanensis Yang, Yang \& Li, 1998

- Hypandrium with long lateral lobes; cercus with basal tubercle. Guizhou, Zhejiang chinensis Yang, 2001

21. Hypandrium symmetrical; cercus with basal tubercle bearing dense long hairs. Yunnan pilifera Yang \& Saigusa, 2001

- Hypandrium asymmetrical; cercus with basal tubercle bearing sparse short hairs. Yunnan yunnanensis Yang \& Saigusa, 2001

22. Halter yellow; hypandrium long and narrow, with V-shaped apical incision. Shaanxi brevifurcata Yang \& Saigusa, 2001

- Halter dark brown with yellow base; hypandrium short and broad, with small, quadrate apical incision. Yunnan

daweishana Yang \& Saigusa, 2001 


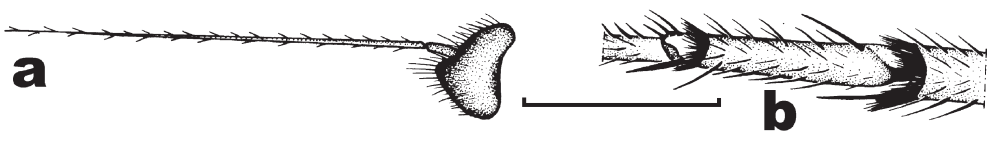

Fig. 1. Nepalomyia beijingensis sp. n., male. a. Antenna, lateral view. b. Hind tarsomere 1, posterior view. c. Wing, dorsal view. d. Genitalia, lateral view. e. Hypandrium, ventral view.

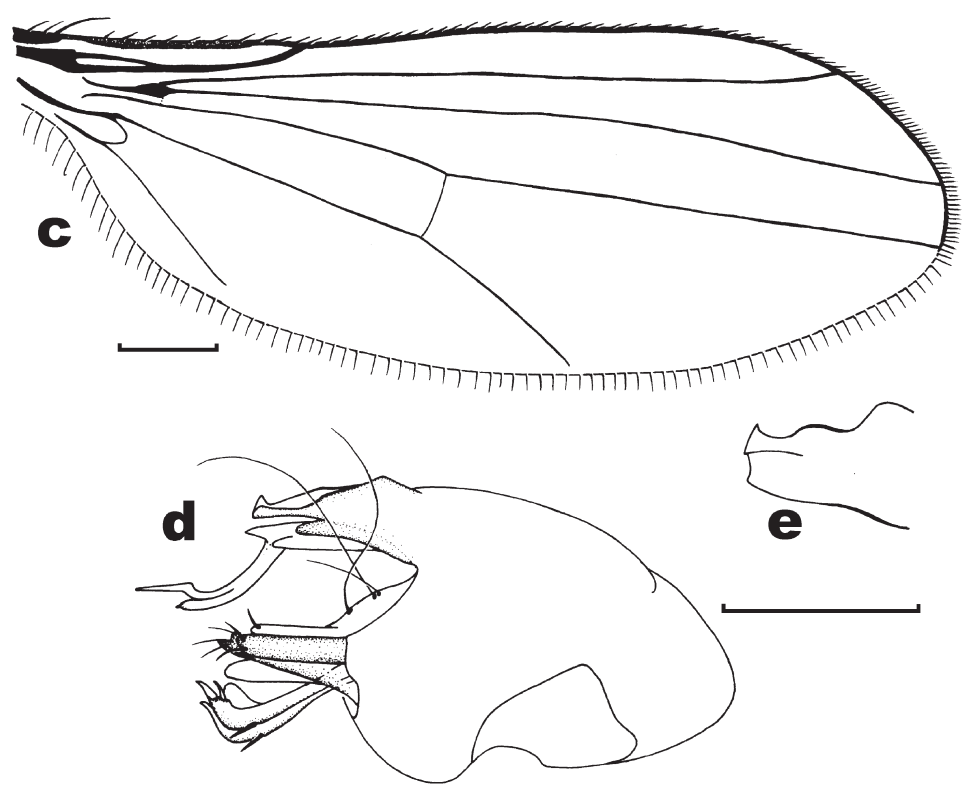

\section{Description of new species}

Nepalomyia beijingensis sp. n. (Fig. 1)

Material examined. Holotype male: China: Beijing, Mentougou, Baihuashan, 13.IX.2003, leg. B. Horvat.

Diagnosis. Thoracic pleura dark brown with brownish-yellow lower portion. 6 strong dc, 3-4 irregularly paired acr. First costal section slightly thickened. Halter dark brown with yellow stem. Male cercus without basal tubercle.

Description. Male. Head dark metallic green with gray-brown pollen; face with pale gray pollen. Hairs and bristles on head black; postocular bristles black, ventral hairs pale. Ocellar tubercle weakly raised, with 2 strong oc and 2 short posterior hairs. Antenna blackish (Fig. 1b); first flagellomere 0.7 times as long as wide, with distinct acute, lower apical angle; arista with basal segment very short. Proboscis and palpus blackish with black hairs.

Thorax dark metallic green with gray pollen; mesonotum and scutellum with gray-brown pollen; pleura dark brown with brownish-yellow lower portion, pteropleuron blackish. Hairs and bristles on thorax black; 6 strong dc, 3-4 irregularly paired acr (reaching the level of the third dc); 1 long h, 1 ph, 1 somewhat short ih, 1 somewhat short su, 1 long anterior and 1 somewhat short posterior npl, 1 somewhat short anterior and 1 long posterior sa, 1 long psa. Scutellum with 2 pairs of bristles, outer pair short and soft. Propleuron with 1 short hair on upper portion and 1 long black bristle on lower portion.

Legs yellow; coxae yellow, mid coxa tinged with brown on posterodorsal angle; mid and hind coxa darker than fore coxa; tarsi brown except tip of tarsomere 5 dark brown. Hairs and bristles on legs black; fore coxa with 6 apical bristles, mid coxa with 1 anterior bristle, hind coxa with 1 outer bristle. Mid and hind femora each with 1 preapical bristle. Fore tibia apically with 1 row of short ad and 1 row of short av, apically with 2 short bristles and brush-like long, pale hairs; mid tibia with 2 ad and 2 pd, apically with 4 bristles ( 2 long and 2 short); hind tibia with 1 row of pd on distal half, apically with 2 long bristles. Fore tarsomeres 1-4 with short, curved, ventral hairs; tarsomere 1 with 2 rows of short v; mid tarsomere 1 with 1 row of short $\mathrm{v}$. Hind tarsomere 1 shorter 


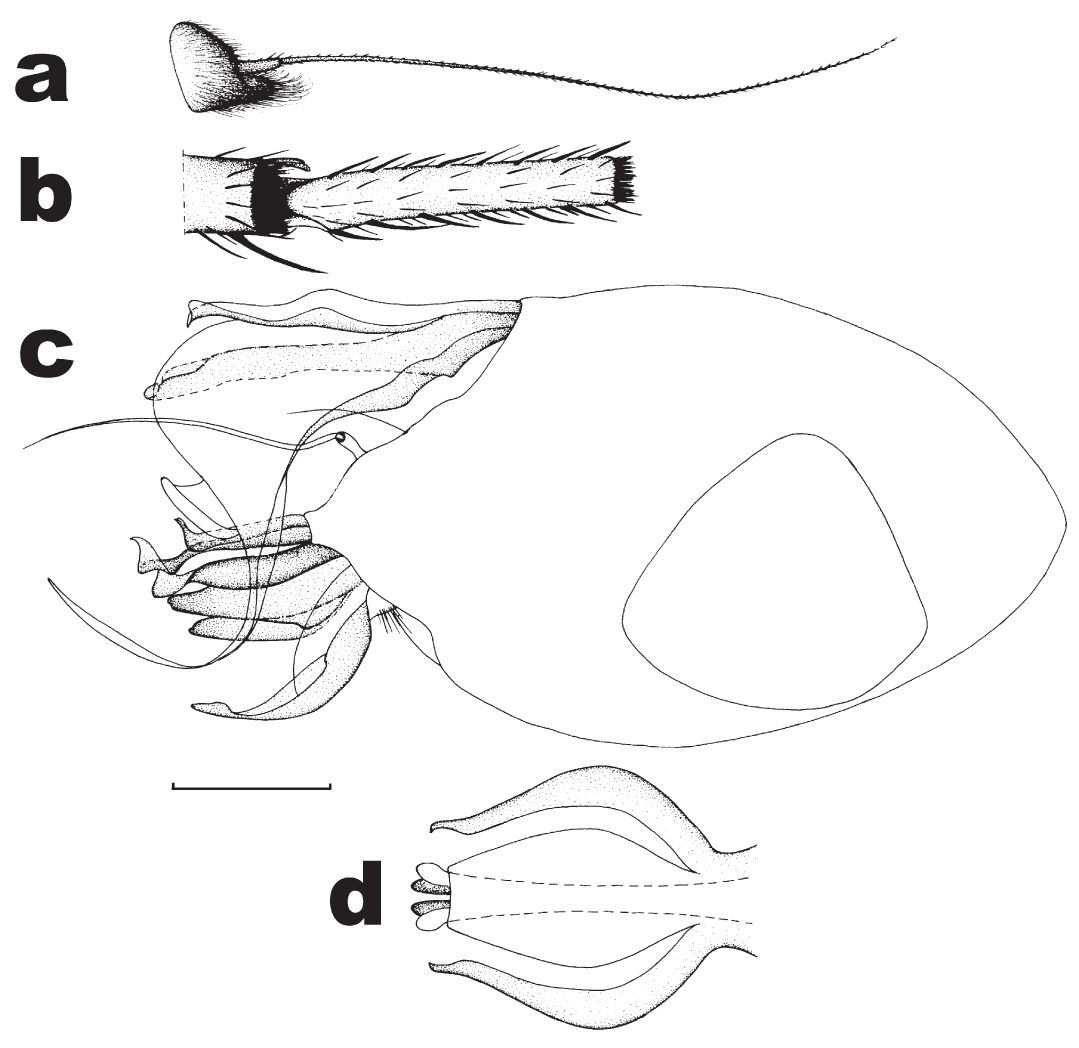

Fig. 2. Nepalomyia ruiliensis sp. n., male. a. Antenna, lateral view. b. Hind tarsomere 1, posterior view. c. Genitalia, lateral view. d. Hypandrium and tip of aedeagus, ventral view. than tarsomere 2, with basal spur on posterior surface curved upward and with $1 \mathrm{v}$ at extreme base, 4 av and 5 pv (Fig. 1e); tarsomere 2 with 2 basal v. Relative lengths of tibia and 5 tarsomeres: LI 2.7 : $2.0: 1.1: 1.1: 0.9: 0.5 ;$ LII $4.5: 2.6: 1.2: 1.1: 0.6$ $: 0.4$; LIII $5.0: 1.3: 2.3: 1.5: 1.0: 0.5$.

Wing hyaline (Fig. 1a), slightly tinged grayish; veins dark brown, first costal section slightly thickened and brownish; R4 +5 and M parallel distally; CuAx ratio 0.34. Squama brown with dark brown margin, with black hairs. Halter dark brown with yellow stem.

Abdomen metallic green with gray-brown pollen. Hairs and bristles on abdomen black; venter with some pale hairs.

Male genitalia (Fig. 1c-d). Surstylus of epandrium with dorsal lobe slightly longer than ventral lobe, acute apically; ventral lobe rather thick with pale hairs apically. Cercus without basal tubercle, with 2 subapical bristle-like processes. Hypandrium short and broad, asymmetrical in ventral view. Aedeagus strongly curved distally, with acute processes.

Length. Body $3.0 \mathrm{~mm}$; wing $2.9 \mathrm{~mm}$.
Female. Unknown.

Remarks. Nepalomyia beijingensis sp. n. is similar to Nepalomyia tuberculosa Yang \& Saigusa from Shaanxi in having a short first flagellomere and asymmetrical hypandrium, but may be separated from the latter by the cercus which lacks a basal tubercle. In N. tuberculosa, the cercus has a thin basal tubercle bearing hairs (Yang \& Saigusa, 2001a).

Distribution. North China (Beijing).

Nepalomyia ruiliensis sp. n. (Fig. 2)

Material examined. Holotype male: Yunnan: Ruili, 750m a.s.1., 3. V. 1981, leg. F. Li. Paratype 1 female: Yunnan: Ruili, 750m a.s.1., 3.V.1981, leg. F. Li.

Diagnosis. First flagellomere about as long as wide; 5 strong dc, 6-7 irregularly paired acr. Hypandrium symmetrical, trifurcate; in ventral view, median lobe broad with truncate tip; in lateral view, lateral lobes strongly curved, with thin apical portions.

Description. Male. Head dark metallic green with gray-brown pollen; face with pale gray pol- 
len. Hairs and bristles on head black; postocular bristles (including ventral hairs) black. Ocellar tubercle weakly raised, with 2 strong oc and 2 short posterior hairs. Antenna brown (Fig. 2a), first flagellomere about as long as wide, with distinct lower, apical angle, with long pale hairs; arista blackish, long, with short blackish hairs, with rather short basal segment. Proboscis brownish with black hairs; palpus black with black hairs.

Thorax pale metallic green with gray pollen; pteropleuron dark. Hairs and bristles on thorax black; 5 strong dc, 6-7 irregularly paired acr; 1 long h, 1 long ph, 1 somewhat short ih, 1 somewhat short su, 1 long anterior and 1 short posterior $\mathrm{npl}, 1$ short anterior and 1 long posterior sa, 1 long psa. Scutellum with 2 pairs of bristles, outer pair weak (1/6 inner pair). Propleuron with 1 black hair on upper portion, with 2 pale hairs and 1 black bristle on lower portion. Legs brownishyellow; coxae dark brown; tarsi distally from tip of tarsomere 1 brown to dark brown. Hairs and bristles on legs black. Fore coxa with 5 apical bristles, mid coxa with 1 anterior bristle, hind coxa with 1 outer bristle. Mid and hind femora each with 1 preapical bristle. Fore tibia apically with 3 short bristles; mid tibia with 2 ad, 2 pd, and $1 \mathrm{av}$, apically with 4 bristles; hind tibia with 1 ad, $2 \mathrm{pd}$, apically with 3 bristles. Hind tarsomere 1 shorter than tarsomere 2, with broad, basal spur on posterior surface curved upward, and with $6 \mathrm{v}$ (Fig. 2d). Relative lengths of tibia and 5 tarsomeres LI $3.1: 2.5: ?: ?$ : ? : ?; LII $2.8: 2.0$ : $1.2: 1.0: 0.7: 0.4$; LIII $5.0: 1.1: 1.6: 1.4: 0.9$ : 0.4 .

Wings hyaline, slightly tinged with grayish; veins dark brown, R4+5 and M1 parallel distally. $\mathrm{CuAx}$ ratio 0.6. Squama brown with black margin, with black hairs. Halter dark yellow.

Abdomen pale metallic green, with graybrown pollen; hairs and bristles black.

Male genitalia (Fig. 2b). Epandrium rather thick, with finger-like apical lateral process bearing 1 very long bristle. Surstylus with thick dorsal lobe bearing subapical lateral incision; middle lobe thick and curved at apex; ventral lobe slender and straight, with curved apex. Hypandrium (Fig. 2c) symmetrical, trifurcate; median lobe somewhat rounded, with truncate apex in ventral view; lateral lobe long with acute apex in ventral view, broad with long thin apex in lateral view. Cercus with black hairs basally, apically with long, curved process. Aedeagus rather thick, with narrow apex in lateral view, with 2 small round processes in ventral view.

Length. Body $3.3 \mathrm{~mm}$; wing $3.6 \mathrm{~mm}$.

Female. Body length $3.2 \mathrm{~mm}$; wing length 3.5 $\mathrm{mm}$. Similar to male.

Remarks. Nepalomyia ruiliensis $\mathrm{sp}$. $\mathrm{n}$. is similar to Nepalomyia pallipilosa Yang \& Saigusa in having the symmetrical, trifurcate hypandrium, but may be separated from the latter by the first flagellomere about as long as wide, and hypandrium without marginal denticles. In $N$. pallipilosa, the first falgellomere is 0.6 times as long as wide, and the hypandrium has distinct marginal denticles (Yang \& Saigusa, 2001b).

Distribution. South China (Yunnan).

\section{Discussion}

China belongs faunistically to two zoogeographical realms: the Palaearctic and the Oriental. Seven zoogeographical regions of China have been designated by Zhang (1998): North-East China, North China, Neimeng-Xinjiang, Qinghai-Xizang, South-West China, Central China and South China. The first 4 regions belong to the Palaearctic Realm, while the latter 3 regions belong to the Oriental Realm.

Distribution of Chinese Nepalomyia species is concentrated in the Oriental Realm. There are 15 species from South China Region and 10 species from Central China Region. One species, $N$. chinensis Yang, is widely distributed in both South China Region and Central China Region. Undoubtedly South China Region is the center of diversity for the Chinese Nepalomyia.

Acknowledgements. The authors wish to thank Dr. Bodgan Horvat (Ljubljana) and Fasheng Li (Beijing) for collecting the specimens and help in many ways. This study was supported by the National Natural Science Foundation of China (No. 30225009).

\section{References}

Dyte, C. E. 1975: Family Dolichopodidae. In: Delfinado, M. D. \& Hardy, D. E. (eds.), A Catalog of the Diptera 
of the Oriental Region 2: 212-258. - The University Press of Hawaii, Honolulu. 459pp.

Hollis, D. 1964: On the Diptera of Nepal (Stratiomyidae, Therevidae and Dolichopodidae). - Bull. Br. Mus. (Nat. Hist.) Ent. 15: 83-116.

Negrobov, O. P. 1984: The genera of the family Dolichopodidae (Diptera), new for the faunas of $\mathrm{Pa}$ laearctic and USSR. - Zool. Zhur. 63(7): 1111-1115.

Negrobov, O. P. 1991: Family Dolichopodidae. In: So , \& Papp, L. (eds.), Catalogue of Palaearctic Diptera 7: 11-139. - Akademiai Kiado, Budapest. 291pp.

Robinson, H. 1964: A synopsis of the Dolichopodidae (Diptera) of the southeastern United States and adjacent regions. - Misc. Publ. Ent. Soc. Am. 4: 103-192.

Robinson, H. \& Vockeroth, J. R.1981: Dolichopodidae. In: McAlpine, J. F. et al. (eds.), Manual of Nearctic Diptera, 1: 625-639. - Research Branch Agriculture Canada Monograph No.27. 674pp.

Runyon, J. B. \& Hurley, R. L. 2003: Revision of the Nearctic species of Nepalomyia Hollis (= Neurigonella Robinson) (Diptera: Dolichopodidae: Peloropeodinae) with a world catalogue. - Ann. Ent. Soc. Am. 96(4): 403-414.

Van Duzee, M. C. 1914: New species of North American Dolichopodidae (Dipt.). — Ent. News 25: 433-443.
Yang, D. 2001: Diptera: Dolichopodidae. In: Wu, H. \& Pan, C. (eds.), Insects of the Tianmushan Mountain: 428-441. - Science Press, Beijing. 764pp. [In Chinese].

Yang D. 2002: Diptera: Therevidae, Dolichopodidae. In: Huang, F. (ed.), Forestry Insects of Hainan: 741-749. - Science Press, Beijing. 1064pp. [In Chinese].

Yang, D. \& Saigusa, T. 2000: New and little known species of Dolichopodidae from China (VII): Diptera from Emei Mountain (2). - Bull. Inst. Royal Sci. Nat. Belg. Ent. 70: 219-242.

Yang, D. \& Saigusa, T. 2001a: The species of Neurigonella from China (Diptera: Empidoidea: Dolichopodidae). - Ann. Soc. Ent. Fr. 37: 375-392.

Yang, D. \& Saigusa, T. 2001b: New and little known species of Dolichopodidae from China (XI). - Bull. Inst. Royal Sci. Nat. Belg. Ent. 71: 237-256.

Yang, D., Yang, C. \& Li, F. 1998: Three new species of Dolichopodidae from Henan (Diptera). - In: Shen, X. \& Shi, Z. (eds.), The Fauna and Taxonomy of Insects in Henan 2: 81-85. China Agricultural Scientech Press, Beijing. 368 pp. [In Chinese].

Zhang, Y. Z. 1998. The second revision of zoogeographical regions of China. - Acta Zootaxon. Sin. 23 (suppl.): 207-222. [In Chinese]. 